\title{
Characterization of the spatial distribution of irradiance and spectrum in concentrating photovoltaic systems and their effect on multi-junction solar cells
}

\author{
M. Victoria*, R. Herrero, C. Domínguez, I. Antón, S. Askins and G. Sala \\ Instituto de Energia Solar, Universidad Politécnica de Madrid, Ciudad Universitaria, 28040 Madrid, Spain
}

\begin{abstract}
The irradiance and spectral distribution cast on the cell by a concentrating photovoltaic system. typically made up of a primary Fresnel lens and a secondary stage optical element, is dependent on many factors, and these distributions in tum influence the electrical performance of the cell. In this paper, the effect of spatial and spectral non-uniform irradiance distribution on multi-junction solar cell performance was analyzed using an integrated approach. Iradiance and spectral distributions were obtained by means of ray-tracing simulation and by direct imaging at a range of cell-to-lens distances. At the same positions, $l-V$ curves were measured and compared in order to evaluate non-uniformity effects on cell performance. The procedure was applied to three different optical systems comprised a Fresnel lens with a secondary optical element consisting of ejther a pyramid. a dome, or a bare cell. Copyright $(92011$ John Wiley \& Sons. Lid.
\end{abstract}

KEYWORDS

multi-junction solar cells; concentrators; ray tracing; non-unifonnity; $K V$ curve

* Correspondence

M. Victoria, Instituto de Energía Solar, Universidad Politecnica de Madrid, Ciudad Universitaria, 28040 Madrid, Spain.

E-mail: marta.victoria@ies-def.upm.es

Received 30 April 2011; Revised 5 August 2011; Accepted 11 August 2011

\section{INTRODUCTION}

Multi-junction (MJ) solar cells have the highest conversion efficiency of any photovoltaic device, wilh measured values reaching up to $43.5 \%$ [1]. These cells produce maximum efficiency values at a certain irradiance level, a certain spectnum (usually ASTM G173-3 AM1.5D), and uniform illumination. However, when MJ solar cells are used in a particular concentrating photovoltaic (CPV) system. the illumination conditions are different from the reference ones, resulting in an efficiency decrease.

Most concentrating optics cause flux distributions that are spatially non-uniform both in absolute irradiance and spectral content. Some cell areas are illuminated at a higher irradiance level than others, translating into an increase in effeclive series resistance and, therefore, a decrease in fill factor $(F F)$. At the same time, other cell areas are very poorly illuminated, or even dark. Hence, those areas will work as dark diodes. contributing only with recombination current but not with photogeneration. In addition. if the peak irradiance generates a current that exceeds the tunnel junction current limis, and the lateral resistance is not negligible, the efficiency will dramatically decrease. Both effects, the loss of FF due to effective series resistance increase and the possibility of surpassing the tunnel limit, have been and are being investigated by different research groups worldwide. Some authors have created more realistic simulations of MJ solar cells using disLributed models [2-7]. Ohers have used simulation to predict the tunnel junction current limit and detemine whether current-spreading phenomena are significant enough to prevent an efficiency decrease or not [8-13].

Spectral non-uniformities, where the spectral content of the flux varies across the cell, are inherent to refractive CPV systems because of the optical transfer function and chromatic aberration in the optics. This spectral variation can signiticantly decrease the current generated by one of the subcells (and consequently the total current passing through the multiple series-connected subcells). A solar cell that is middle limited under the reference spectrim can be top limited inside a particular CPV system under the same illumination or, more likely. can have some areas top limited whereas others are middle limiled. In this case. the limiting subcell will be determined by lateral current flow in the layers connecting the subcells [4]. 
Both types of spatial irradiance non-uniformities, both spectral and absolute, are highly sensitive to the receiver-to-primary lens distance and, as will be shown here, an experimental determination of the optimum cell receiver position is highly recommended for any new concentrator design.

Different approaches have been followed to study and quantify the effects of non-uniform irradiance distribution on MJ solar cells. Leutz [14] and James [7] used ray-tracing simulations to predict the irradiance distribution for different light colors when using a Fresnel lens with and without a secondary optical element (SOE). Miñano [15] has also shown how imaging systems can cause these non-uniformities and how they can be smoothed by using non-imaging optics and Köhler integration. In an earlier work, the authors of this paper have presented the simulated profiles obtained by adding different refractive and reflective SOEs to a Fresnel lens [16]. These resulting irradiance profiles can be used to feed the solar cell-distributed models previously mentioned and predict cell performance. However, these approaches have uncertainties due to the fact that ray tracing predicts irradiance peaks higher than the ones taking place in reality if optical surfaces and bulk are considered ideal. To overcome that, the optical performance of surfaces and bulk material, which are very dependent on material and manufacturing process, must be accurately known.

A more experimental approach was followed by Katz [17] using a fiber glass to create an extremely high-illuminated area on a solar cell. Although the solar cell performance declines for peak irradiances higher than 10000 suns, it is not clear that real CPV systems will produce such irradiance distribution (small-area sharp peaks).

We recently introduced another approach based on using simulated irradiance profiles to generate high resolution masks for imposing a certain uniformity map and comparing the $I-V$ curve of the cell covered with the mask, with the $I-V$ curve under uniform illumination to calculate the $F F$ decrease [18]. The main drawback of this method is that masks only reproduce spatial non-uniformity, neglecting the spectral one. Finally, Cotal [19] and Kurtz [20] measured the cell under the optical system (point-focus flat
Fresnel and linear dome Fresnel, respectively) and obtained the efficiency losses for different cell positions.

In this paper, an integral approach was followed, comparing results from ray-tracing simulations, charge-coupled device (CCD)-based camera measurements, and directly measured $I-V$ curves. Three CPV systems, all with the same geometrical concentration of $625 \mathrm{X}$, were studied. The systems comprised a Fresnel lens and either a bare cell, a dielectric truncated pyramid SOE, or a refracting dome SOE (Figure 1). An MJ solar cell of the same size $\left(1 \mathrm{~cm}^{2}\right)$ and technology, which had been previously calibrated under homogeneous concentrated flash light, was used in each case. The SOE exit is slightly smaller than the cell active area to avoid spilled light beyond the cell perimeter. First, the optical systems were simulated using Monte-Carlo ray tracing, using a source which spectral and angular characteristics were that of the sun. From this simulation, considering spectral flux distribution across the cell and the spectral response of each subcell, the distributed photogenerated current for each subcell was calculated. Then, irradiance distributions were measured using a CCD camera and adequate filters. CCD measurements for different receiverto-primary lens distances were correlated with simulated results. Finally, the $I-V$ curves of the systems for different receiver positions were measured, and the variation of the electrical parameters is analyzed and compared with the irradiance distribution that caused this variation.

\section{SIMULATIONS AND MEASUREMENTS DESCRIPTION}

\subsection{Ray-tracing simulations}

Ray-tracing simulations can be very useful to predict optical performance and, in particular, the irradiance profiles produced by different CPV systems. In order for the simulations to be as realistic as possible, one must consider the sun angular size and the transmittance of all the materials comprising the system.

\section{Fresnel Lens}
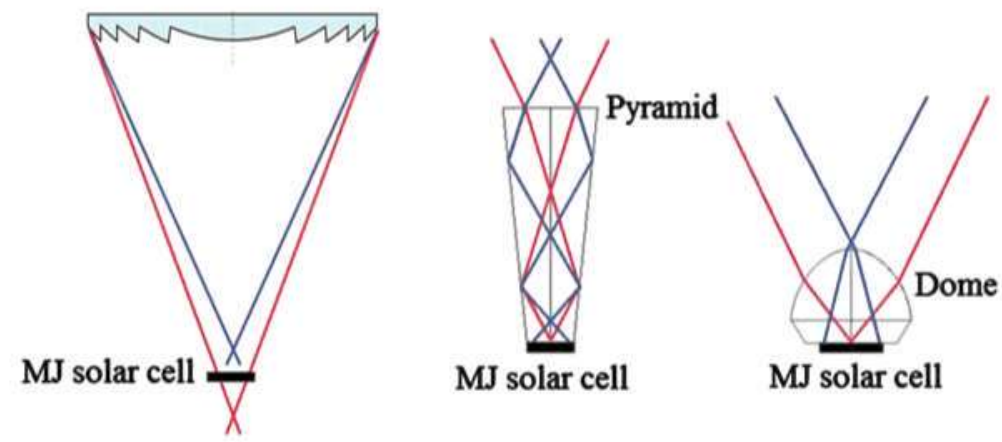

Figure 1. Optical systems studied in this paper comprised a Fresnel lens and three different receivers: pyramid secondary optical element (SOE) (center), dome SOE (right), and bare cell (left). MJ, multi-junction. 
Instead of using the reference spectrum, $B(\lambda)$, for the emitting source, an artificial spectrum $S(\lambda)$ was used in the simulations. $S(\lambda)$ results from multiplying the MJ solar cells spectral response $S R(\lambda)$ times the reference spectrum $B(\lambda)$.

$$
S(\lambda)=B(\lambda) S R(\lambda)
$$

Therefore, instead of predicting irradiance distribution over the cell, the simulation output represents the photogenerated current distribution for each subcell, making it possible to determine which subcell is limiting in any part of the cell. The true cell-limiting mode is strongly influenced by the sheet resistance of the layers connecting subcells. For each connection, the middle layer resistance comprised the top subcell base sheet resistance, the tunnel junction sheet resistance, and the bottom subcell emitter sheet resistance. A detailed analysis can be found in Kurtz and O'Neill [4], but the important conclusion is that those parameters can make the effect of chromatic aberration on cell performance vary from significant to negligible.

Certain optical non-idealities were neglected in the simulations. The most important is scattering, because optical surface roughness is very dependent on the optical material and manufacturing process and difficult to simulate. Defects, misalignment errors, and dust can also contribute to scattering in a real system. The addition of scattering would significantly smooth irradiance peaks predicted by simulations, so the results presented here can be considered as worst case.

We have rendered a ray-tracing simulation using the Monte-Carlo method with 1 million rays for every optical system and for every receiver-to-primary lens distance. The estimated error for each of the $40 \times 40$ nodes in the receiver that simulate the solar cell entrance was less than $5 \%$.

\subsection{Irradiance profile characterization}

An experimental method to measure the irradiance profiles using a CCD camera has been developed and presented elsewhere [21]. The CPV system under study was illuminated by the Helios 3198 solar simulator (Soldaduras Avanzadas, Puertollano, Spain) [22], which provides highly collimated light that is spectrally matched to AM1.5D. The solar cell was substituted by a translucent Lambertian diffusing surface, and the irradiance distribution at that plane was imaged with a CCD camera. By using adequate low-pass or highpass filters, the irradiance profile of the spectral band seen by each subcell could be separately recorded. SR differences between the camera and the subcells (Figure 2) may introduce some errors mainly due to the difference in the SR limits for each case (differences in the absolute SR values can be neglected as the camera is not used as an absolute irradiance level sensor). As in the simulations, the actual irradiance was weighted by the spectral response of the CCD camera sensor (plus the filters), and therefore represents an estimation of the photogeneration rather than irradiance. Although it is difficult to obtain an absolute irradiance value using the CCD camera as its sensitivity depends on the exposure time and lens aperture, the relative irradiances at different pixels for the same image are valid. Peak-to-average ratios (PAR) for top and middle subcells at each cell position were compared with the simulated values. In general, PAR measured values are lower than the simulated ones due to the scattering mentioned above.

The irradiance profile seen by the bottom subcell cannot be measured because the silicon sensor in the CCD camera cannot detect light at the wavelengths this subcell is sensitive to. However, for the majority of optical materials, dispersion is far more significant at lower wavelengths than at higher ones, and so the bottom cell irradiance profile is far more similar to middle cell irradiance profile than middle is to top. In addition, for the semiconductors currently used to manufacture MJ solar cells, the bottom subcell has an excess of photogenerated current, meaning that MJ solar cells are usually either top or middle limited. For these reasons, and for the sake of clarity, the influence of the bottom subcell is neglected for the

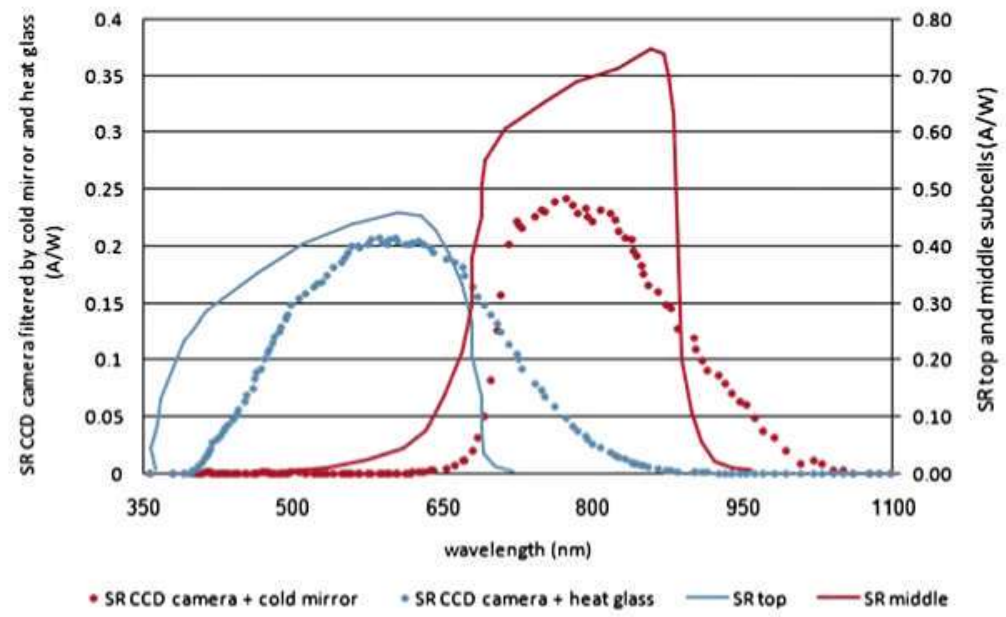

Figure 2. Spectral response of the charge-coupled device (CCD) camera silicon sensor filtered by cold mirror and heat glass (solid lines) to simulate spectral response of middle and top subcells (dots). PAR, peak-to-average ratio. 
rest of this paper, as we turn to the question of how these irradiance profiles affect the solar cell performance.

\subsection{Electrical measurements}

To come full circle and analyze cell performance under spectral and spatial non-uniform distributions, the electrical performance at different receiver-to-primary lens distance was measured in the solar simulator. At every position, an $I-V$ curve was recorded under different irradiance levels (direct normal irradiance (DNI) from 600 to $1000 \mathrm{~W} / \mathrm{m}^{2}$ ) and different spectral distributions. The electrical parameters of short-circuit current $\left(I_{\mathrm{sc}}\right)$, fill factor $(F F)$, and efficiency () were then calculated from the $I-V$ curve.

Throughout the paper, a figure of merit is used for describing the spectrum: the Spectral Matching Ratio $(S M R)$, which is defined as the ratio between the top and middle subcell photogenerated currents under the spectrum in study, divided by that ratio under the reference spectrum, defined as

$$
S M R(A M 1.5 D)=\frac{i_{L, \text { top }}\left(E_{\text {simulator }}\right) / I_{I_{L, \text { mididle }}}\left(E_{\text {simulator }}\right)}{i_{L, \text { top }}^{*}(A M 1.5 D) / i_{L, \text { middic }}^{*}(A M 1.5 D)}
$$

where $I_{\mathrm{L}}(E)$ represents the photocurrent of a subcell (top or middle) when illuminated with a particular spectral irradiance distribution $E$ under the simulator (which in turn depends on the irradiance level). $I_{\mathrm{L}}^{*}(\mathrm{AM} 1.5 \mathrm{D})$ stands for the photoresponse of a subcell under the reference spectrum. $S M R$ is an indicator of how blue-shifted $(S M R>1)$ or red-shifted $(S M R<1)$ the light is with respect to the reference spectrum. The values of $S M R$ for the measurements presented in this article, between 0.86 and 0.96 are representative of values that may be obtained outdoors yearlong in, for example, Madrid (continental climate, latitude $40^{\circ}$ ), whereas higher values of 1.0 and beyond are only obtained in the summer months.
An important point about the experimental method used is that the SMR and the irradiance are varied simultaneously, because of intrinsic properties of the light source: the spectrum shifts from blue to red during the flash pulse decay. This must be kept in mind when interpreting results, as the electrical behavior is affected by both irradiance and spectrum. However, it should be noted that this is also representative of the change in solar irradiance and spectrum throughout the day in most locations: bluer spectrums correspond with a higher DNI and vice-versa. For example, in Figure 3 we can see the variation of $S M R$ both on a day near the spring equinox in Madrid and in the simulator.

It will be seen that the relative maximum of $I_{\mathrm{sc}}$ versus focal distance is obtained at the location where top and middle subcells are current matched. $F F$ is influenced simultaneously by the changing irradiance, spectrum, and spatial non-uniformities. When a cell is uniformly illuminated, $F F$ is strongly influenced by current matching in the cell. If the short-circuit current is kept constant, the minimum $F F$ is obtained when both subcells are matched $[23,24]$. The measured values of $\eta$, determined from $I_{\mathrm{sc}}$ and $F F$, are shown in the same figures. As the main objective of this paper is to analyze how the concentrator performance depends on the primary-receiver position and the spectral distribution, $\eta$ and $I_{\mathrm{sc}}$ are presented normalized by their maximum in each system. $F F$ is a relative value by definition and so is not normalized.

\section{EXPERIMENTAL}

\subsection{Fresnel lens with bare cell}

The irradiance profile produced by a classic point-focus Fresnel lens is well known, so this system can be useful to validate the entire procedure. As is expected, blue light (used by the top subcell) has a PAR maximum when the cell is nearer the lens than its design position. On the other hand, the PAR is maximized for red light (used by middle subcell) at focal distances higher than design (Figure 4).

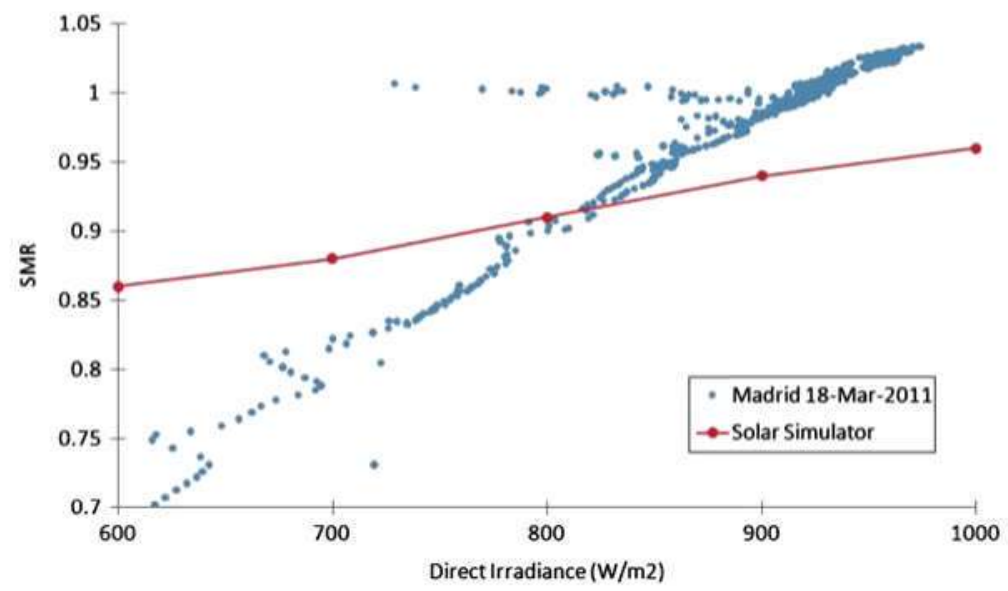

Figure 3. Comparison of spectrum dependence on direct normal irradiance for indoor and outdoor measurements. 


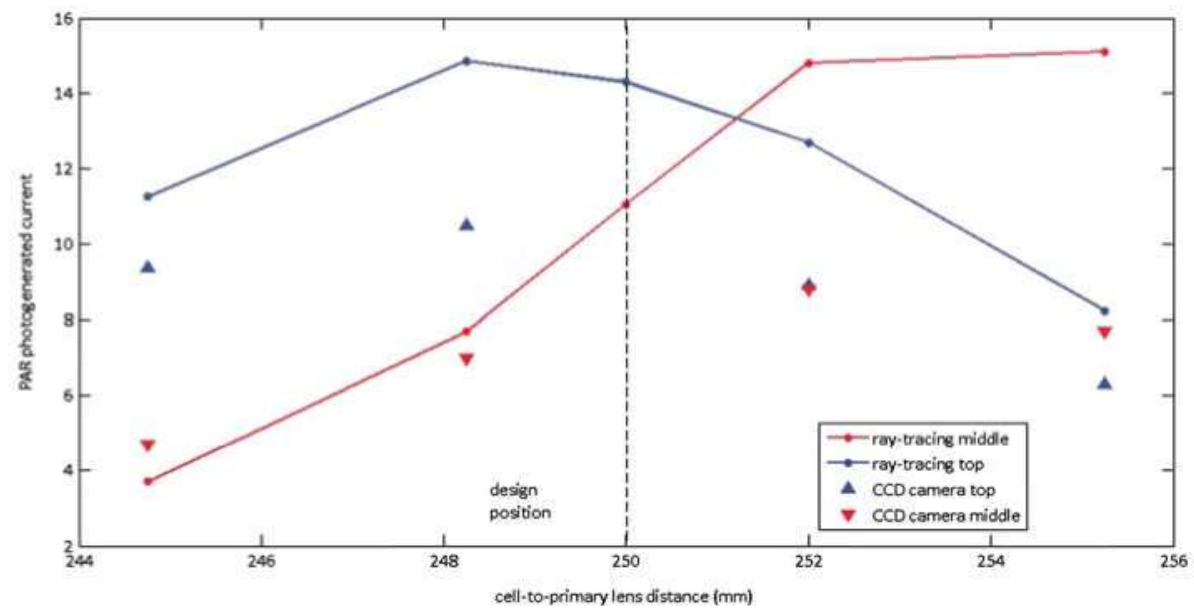

Figure 4. Irradiance profiles peak-to-average ratios caused by a Fresnel lens. Continuous lines for simulated predictions (blue for the top and red for the middle subcell). Triangles for the charge-coupled device measurements (blue for the low-pass filer corresponding to the top subcell and red for the high-pass filter corresponding to the middle subcell).FF, fill factor; SMR, spectral matching ratio; SOE, secondary optical element.

Ray-tracing simulations with ideal optical surfaces predicted a maximum PAR of 15 . CCD measurements showed similar overall tendencies but predicted lower irradiance peaks, with a maximum of 10 .

In Figure 5 the results from the ray-tracing simulations are shown. For each cell-to-lens distance, the spatial distribution of the current photogenerated by the top subcell is shown in the top row and that of the current photogenerated by the middle subcell is shown on the second row. The third row represents the ratio between the two of them (i.e., current matching). In this last row, the zones where the ratio is lower than 1 (blue) will be top limited,
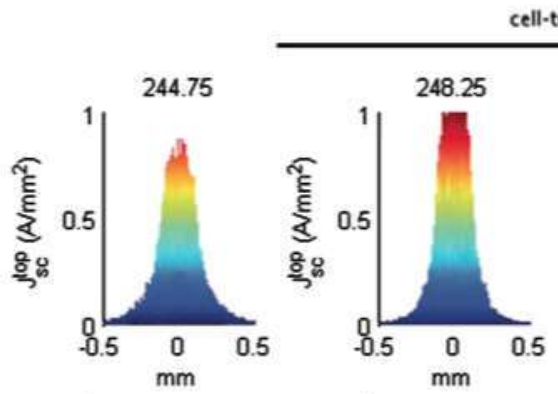

cell-to-lens distance increases
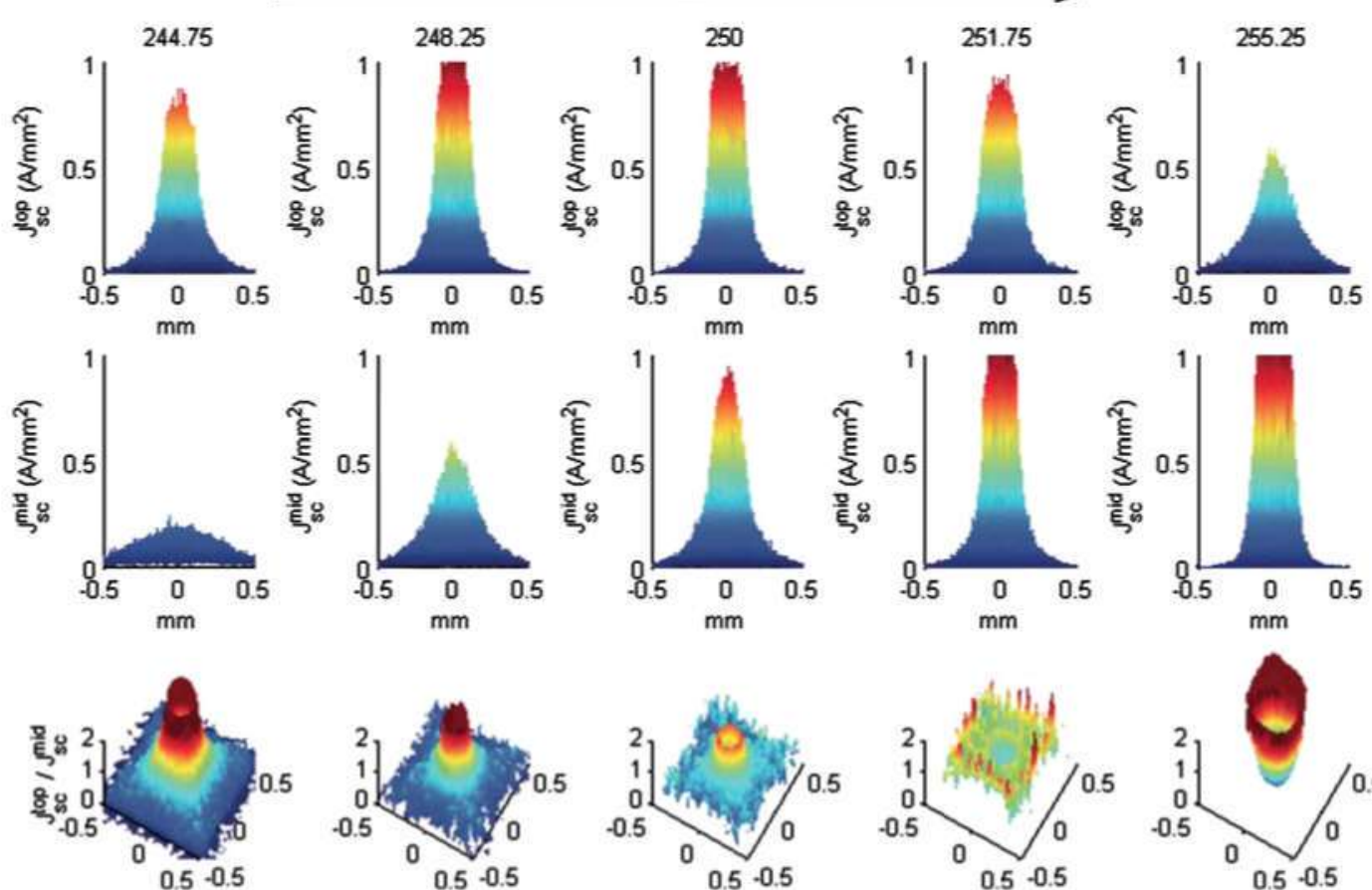

Figure 5. Fresnel lens plus bare-cell system. Top subcell photogenerated current density (first row) and middle subcell photogenerated current density (second row). Ratio between both is represented in the third row. All the results are for a situation where SMR = 0.96. SMR, spectral matching ratio; FF, fill factor; SOE, secondary optical element. 
whereas those others where the ratio is higher than 1 (red) will be middle limited; zones painted in green are very close to current matching. Using this type of representation, the situations where spectral non-uniformity is significant can be clearly observed.

The representative electrical parameters of a system comprised a bare MJ solar cell and a Fresnel lens, for the same receiver-to-primary lens distances that are simulated by ray tracing, are shown in Figure 6 . The evolution of the $I_{\mathrm{sc}}$ for different positions can be easily understood by comparing Figures 5 and 6 . When the cell was near the lens, there was an excess of blue light that caused the middle subcell to be limiting, then as the cell-to-lens distance was increased and the red-light spot became focused, the top and middle cells became better matched, maximizing $I_{\mathrm{sc}}$. As the cell was moved even farther it became top limited, and $I_{\mathrm{sc}}$ decreased again.

The other variable that strongly influences the system performance is the incident irradiance spectral distribution. As described previously, the irradiance and spectrum were varied simultaneously, and each spectrum is characterized by an $S M R$ value [22]. Figure 6 also shows how, as redder (lower SMR) spectrums are used, the $I_{\mathrm{sc}}$ falls off faster as the receiver-to-primary distance is increased past the optimum, because, with less blue light, the top subcell becomes current limited earlier.

At $S M R=0.96$ and $D N I=1000 \mathrm{~W} / \mathrm{m}^{2}, F F$ is minimized by two effects that occur at the same receiver-to-primary distance: the system is focused, increasing spatial non-uniformity, and the top and middle subcells are current matched. As redder spectrums are used, not only does the $F F$ minimum shift to the left, but the shape of the curve changes. For instance, at $S M R=0.88$ and $700 \mathrm{~W} / \mathrm{m}^{2}$, the $F F$ evolution with position shows a W-shape with two local minima similar to that reported by Cotal [19]. An important conclusion from this result is that at low irradiance values (and low $S M R$ ) the $F F$ remains high and is not very sensitive to cell position as nonuniformities are smoothed by low effective series resistance and top cell is always limiting. However, as irradiance and $S M R$ are increased, $F F$ becomes very sensitive to cell position as significant series resistance make non-uniformities noticeable and limiting subcell strongly depends on cell-to-receiver distance. The optimum focal distance (maximum $\eta$ ) is found at $248.3 \mathrm{~mm}$, which is not coincident with the design position $(250 \mathrm{~mm})$. Therefore, it is highly recommendable to experimentally determine the optimal cell position for any new concentrator design. In this example, assuming the design position, which has a $0.7 \%$ difference with true optimum, would lead to an efficiency decrease of $6.6 \%$.

\subsection{Fresnel lens plus pyramid secondary optical element system}

A dielectric SOE in the form of a truncated pyramid has been proposed as the simplest solution to homogenize irradiance profiles over the cell $[2,25]$. This is seen to be the case: the maximum PAR predicted by simulation is 3 , whereas the maximum PAR measured via imaging is 2
(Figure 7). In addition, the irradiance profile is far less sensitive to cell position than without using an SOE. The important question is then whether uniformity advantages justify the additional cost of adding a pyramid or not.

The homogenizing effect of the pyramid can be clearly observed in Figure 8: the spatial irradiance distribution is very uniform for all the positions studied and for both subcells. This is coherent with the fact that measured $F F$ is in

Fresnel + bare cell
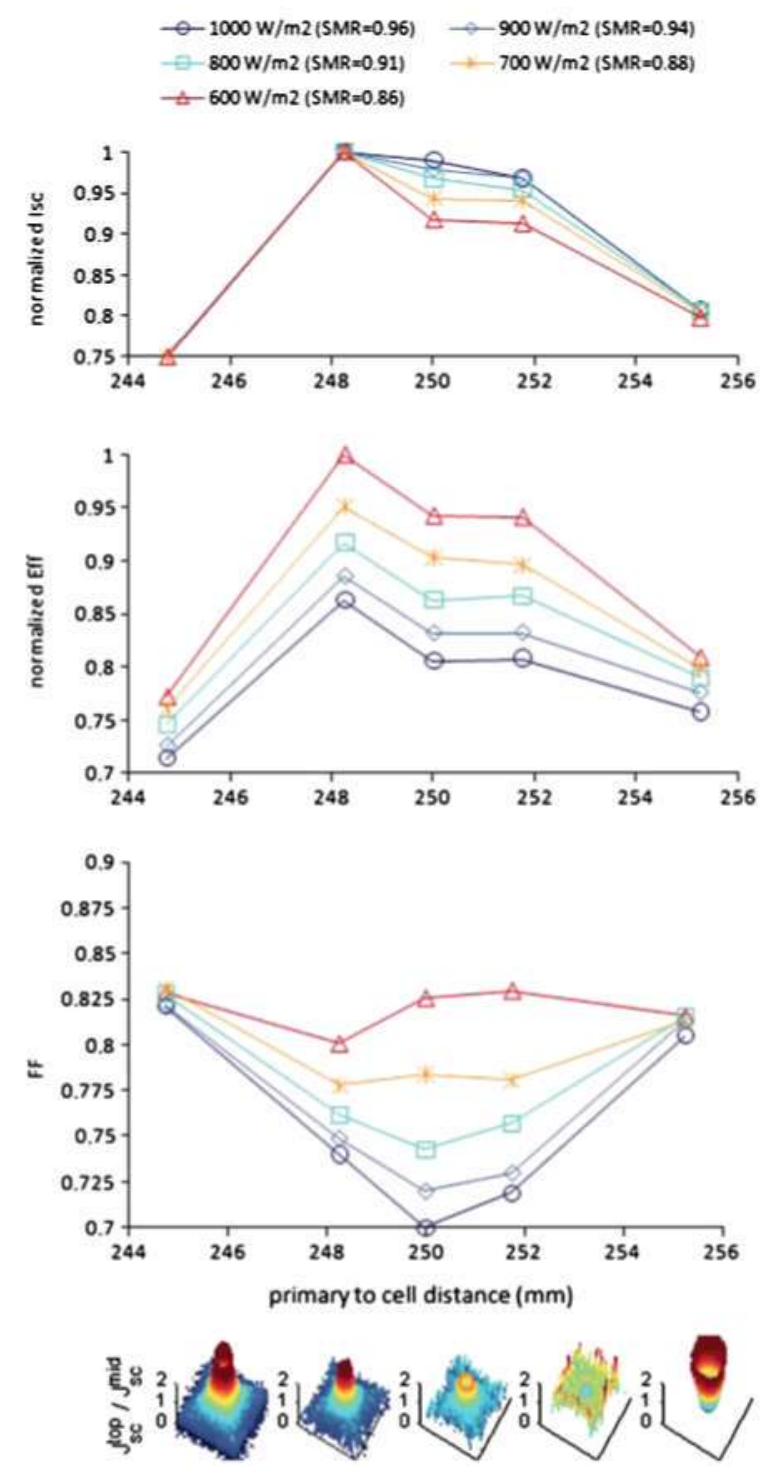

Figure 6. Electrical measurements for the Fresnel concentration system at different primary-to-cell distance. Design position is $250 \mathrm{~mm}$. Plots at the bottom represent ratio between top and middle photogenerated current for each measurement position at $1000 \mathrm{~W} / \mathrm{m}^{2}$ (SMR $=0.96$ ). Ratios higher than 1 (red) indicate middle-limited zones, whereas ratios lower than 1 (blue) indicate top-limited zones. In green areas, top and middle photogenerated current is matched. SR, spectral response; CCD, chargecoupled device. 


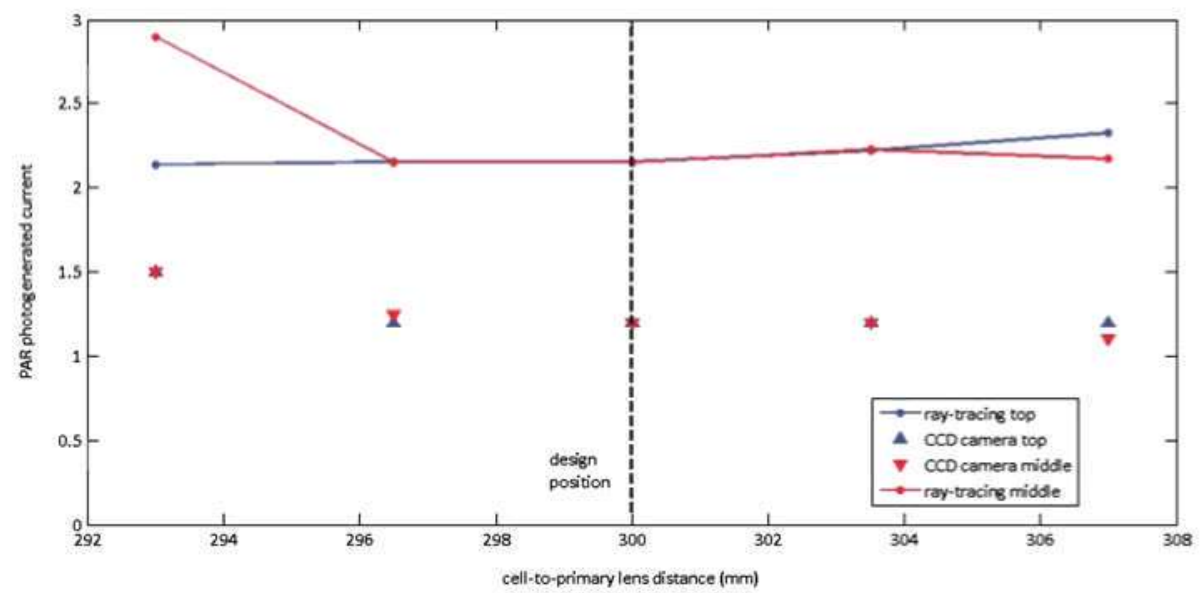

Figure 7. Irradiance profiles peak-to-average ratios caused by a Fresnel lens and a pyramid secondary optical element. Continuous lines for simulated predictions (blue for the top and red for the middle subcell). Triangles for the charge-coupled device measurements (blue for the low-pass filter corresponding to the top subcell and red for the high-pass filter corresponding to the middle subcell). SMR, spectral matching ratio.

general higher than for the Fresnel alone. Furthermore, the ratio between top and middle currents remains close to 1 across the surface of the cell for almost every position. In other words, the optic spatially homogenizes the irradiance distribution spectrally.

Electrical measurements of the system with the pyramid (Figure 9) show a more gradual curve of $I_{\mathrm{sc}}$ with respect to cell-to-lens distance than was seen for the bare-cell measurements. As the spectrum becomes redder, the maximum $I_{\mathrm{sc}}$ shifts to shorter receiver-to-primary lens distances, similar to the previous system. The optimum current matching (i.e., maximum $I_{\mathrm{sc}}$ ) varies slightly with the spectrum, from focal distances of $293 \mathrm{~mm}$ for lower values of $S M R$ to $296 \mathrm{~mm}$ for higher values of $S M R$. Because the spatial irradiance distribution on the cell does not change significantly with position, because of the homogenization, the change in $F F$ depends mainly on current matching between top and middle subcells, without any noticeable difference in behavior for different spectra. Only at the closest position measured, $293 \mathrm{~mm}$, can
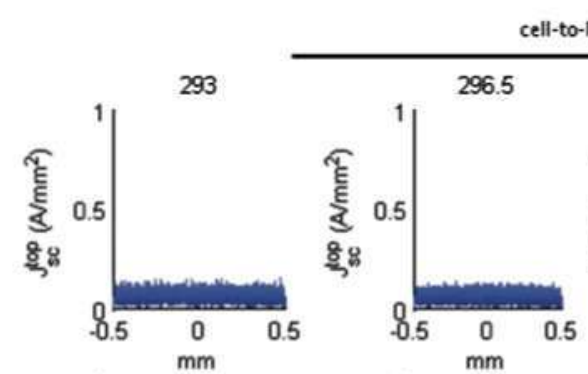

cell-to-lens distance increases
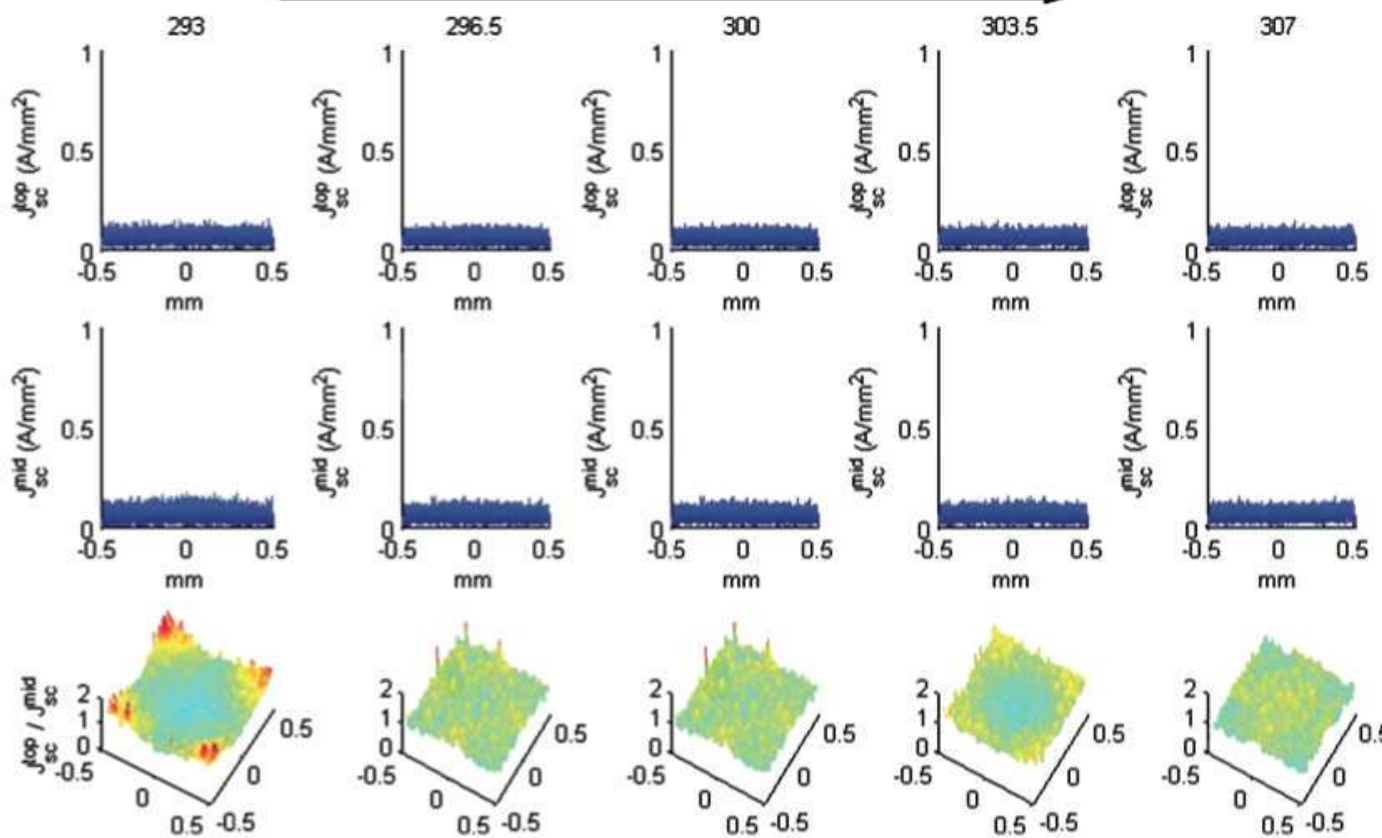

Figure 8. Fresnel lens plus pyramid secondary optical element system. Top subcell photogenerated current density (first row) and middle subcell photogenerated current density (second row). Ratio between both is represented in the third row. All the results are for a situation where $S M R=0.96$. $P A R$, peak-to-average ratio; $C C D$, charge-coupled device. 
a decrease in photogenerated current uniformity be seen, causing a decrease in $F F$ mainly for low $S M R$ values, with little change in $I_{\mathrm{sc}}$.

\subsection{Fresnel lens plus dome secondary optical element system}

Using a dome SOE to homogenize light distribution created by a Fresnel lens was also proposed many years ago
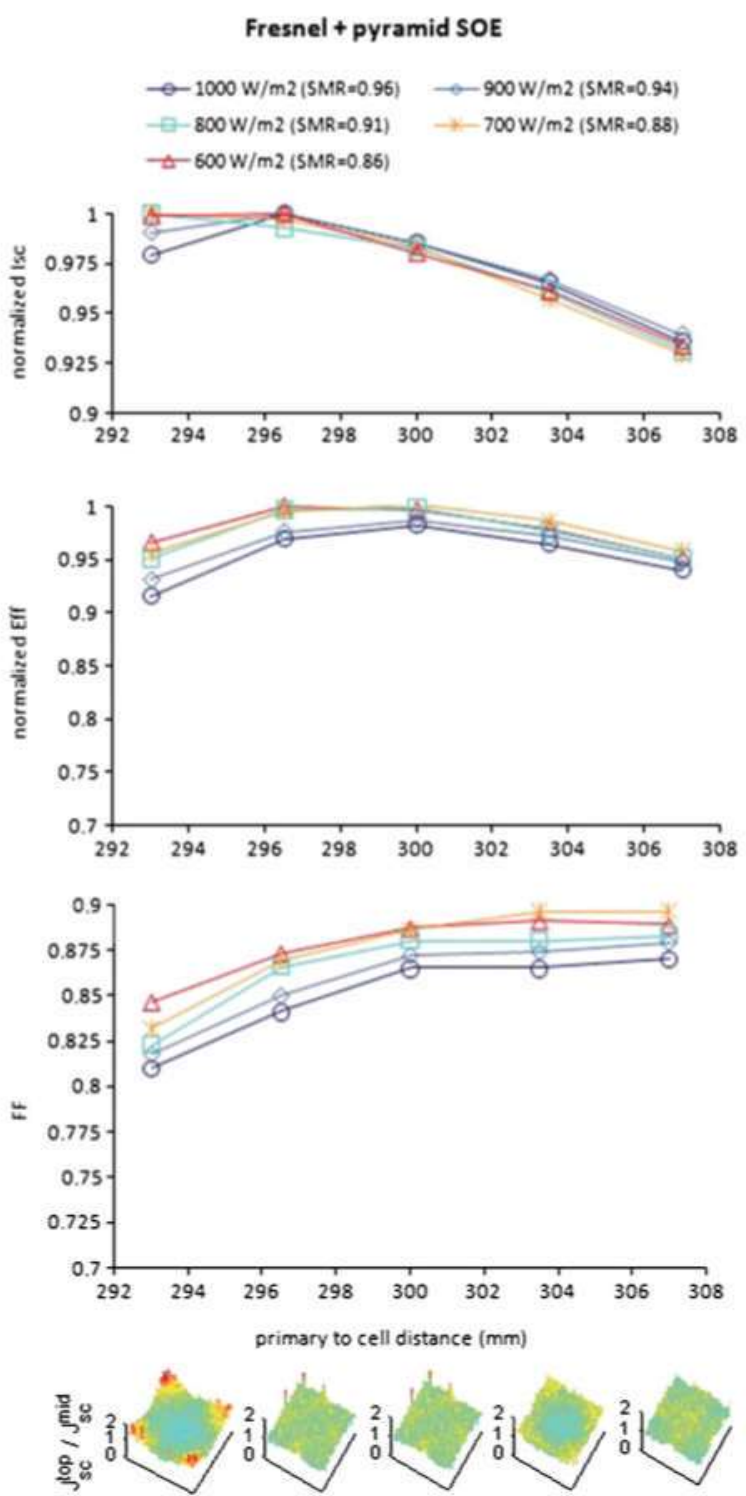

Figure 9. Electrical measurements for the Fresnel+pyramid secondary optical element concentration system at different cell positions. Design position is $300 \mathrm{~mm}$. Plots at the bottom represent ratio between top and middle photogenerated current for each measurement position at $1000 \mathrm{~W} / \mathrm{m}^{2}$ (SMR $=0.96$ ). Ratios higher than 1 (red) indicate middle-limited zones, whereas ratios lower than 1 (blue) indicate top-limited zones. In green areas, top and middle photogenerated current is matched.
$[7,26,27]$. In this proposal, the dome is designed using the Fermat principle under the condition that it images the primary lens on the cell. Hence, as the primary lens is uniformly irradiated by the sun, the cell will be uniformly irradiated by the primary. However, this condition is only true for the design wavelength, whereas at other wavelengths, non-uniform irradiance profiles are obtained. For this effort, we have fabricated a custom dome using optical-grade silicone.

Peak-to-average ratio values obtained when simulating and measuring (Figure 10) are slightly higher than for the system using a pyramid SOE, but much lower than using no SOE at all. For the optimum position, the measured and simulated PAR value is around 3 . When the cell approaches the primary lens, irradiance non-uniformity in the red light increases as the dome is no longer imaging the primary lens on the cell, but has begun to act as a converging lens, as can be clearly observed in Figure 11.

Both ray-tracing simulations and $I_{\mathrm{sc}}$ measurements agree that top subcell is limiting in all the positions. It is possible that this limitation is caused by the transmissivity of silicone. For the longest cell-to-lens distance measured, $268 \mathrm{~mm}$, part of the blue light from the primary does not reach the dome entrance at all, reducing considerably top photogenerated current and, as a consequence, $I_{\mathrm{sc}}$.

Analyzing electrical measurements (Figure 12), we first observe that the $I_{\mathrm{se}}$ maximum occurs at $257 \mathrm{~mm}$, and that this therefore most likely represents the focal distance at which current matching occurs, even though the simulation predicted this match at $268 \mathrm{~mm}$. This discrepancy may be because the dome material spectral transmissivity was not well known. It is observed that $F F$ is at a minimum at the experimentally determined matching position, which also corresponds to the position with the most illumination non-uniformity. At higher focal distances, the $F F$ increases due to both current mismatch in the subcells and decreasing non-uniformity. The latter seems to be the primary cause for the change in $F F$ for cell positions for focal distances between 257 and $265 \mathrm{~mm}$, as the $I_{\mathrm{sc}}$ is almost constant throughout this range.

\section{IRRADIANCE LEVEL AND SPECTRAL DISTRIBUTION SENSITIVITY}

Figure 13 shows $F F$ evolution for different irradiance levels, with correspondingly different spectral distributions, for the three systems at their optimum positions. The three of them show similar tendency; FF decreases as irradiance level increases (and so does effective concentration), and $S M R$ is closer to 1 (so top and middle are closer to match because the system is at its optimum position). However, relative $F F$ variation is far more significant in the bare-cell system than in the system using an SOE, because of the absence of homogenization.

The irradiance levels and SMR values used in these measurements represent real working conditions for a 


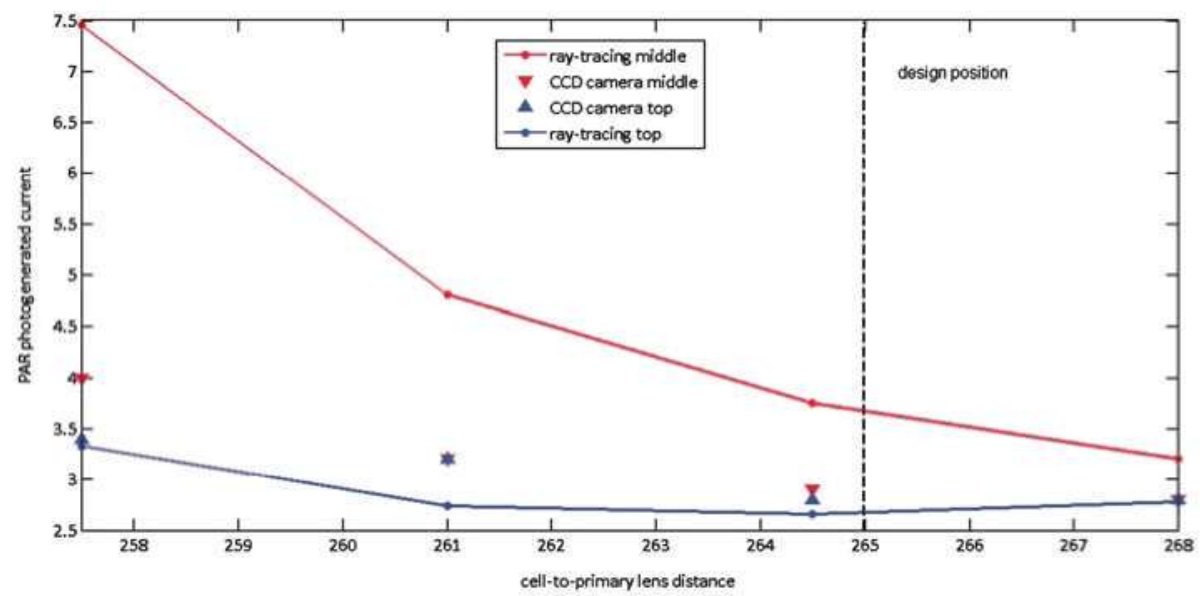

Figure 10. Irradiance profiles peak-to-average ratio caused by a Fresnel lens and a dome secondary optical element. Continuous lines for simulated predictions (blue for the top and red for the middle subcell). Triangles for the charge-coupled device measurements (blue for the low-pass filter corresponding to the top subcell and red for the high-pass filter corresponding to the middle subcell). FF, fill factor; SMR, spectral matching ratio.

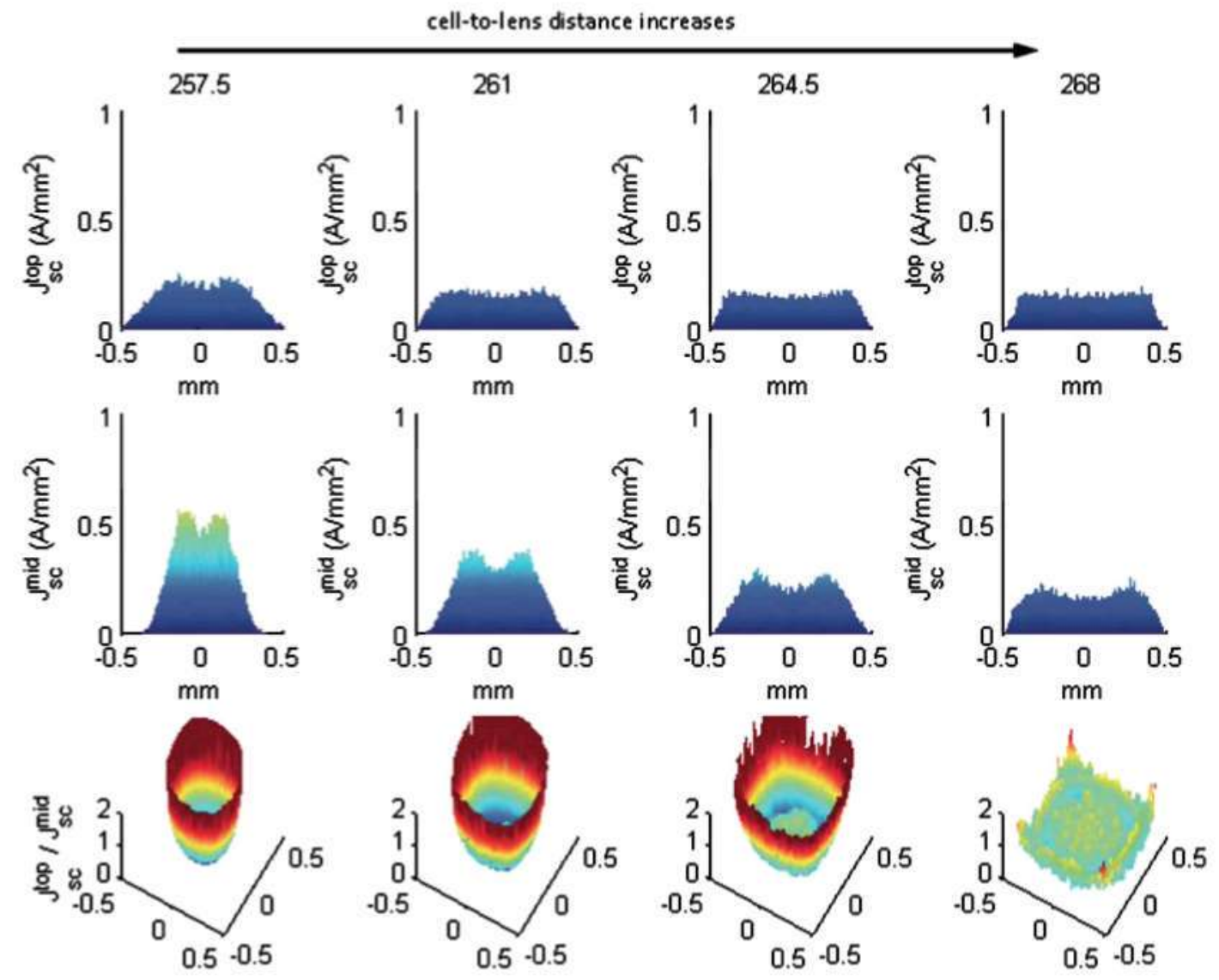

Figure 11. Fresnel lens plus dome secondary optical element system. Top subcell photogenerated current density (first row) and middle subcell photogenerated current density (second row). Ratio between both is represented in the third row. All the results are for a situation where $S M R=0.96$. PAR, peak-to-average ratio; $C C D$, charge-coupled device. 


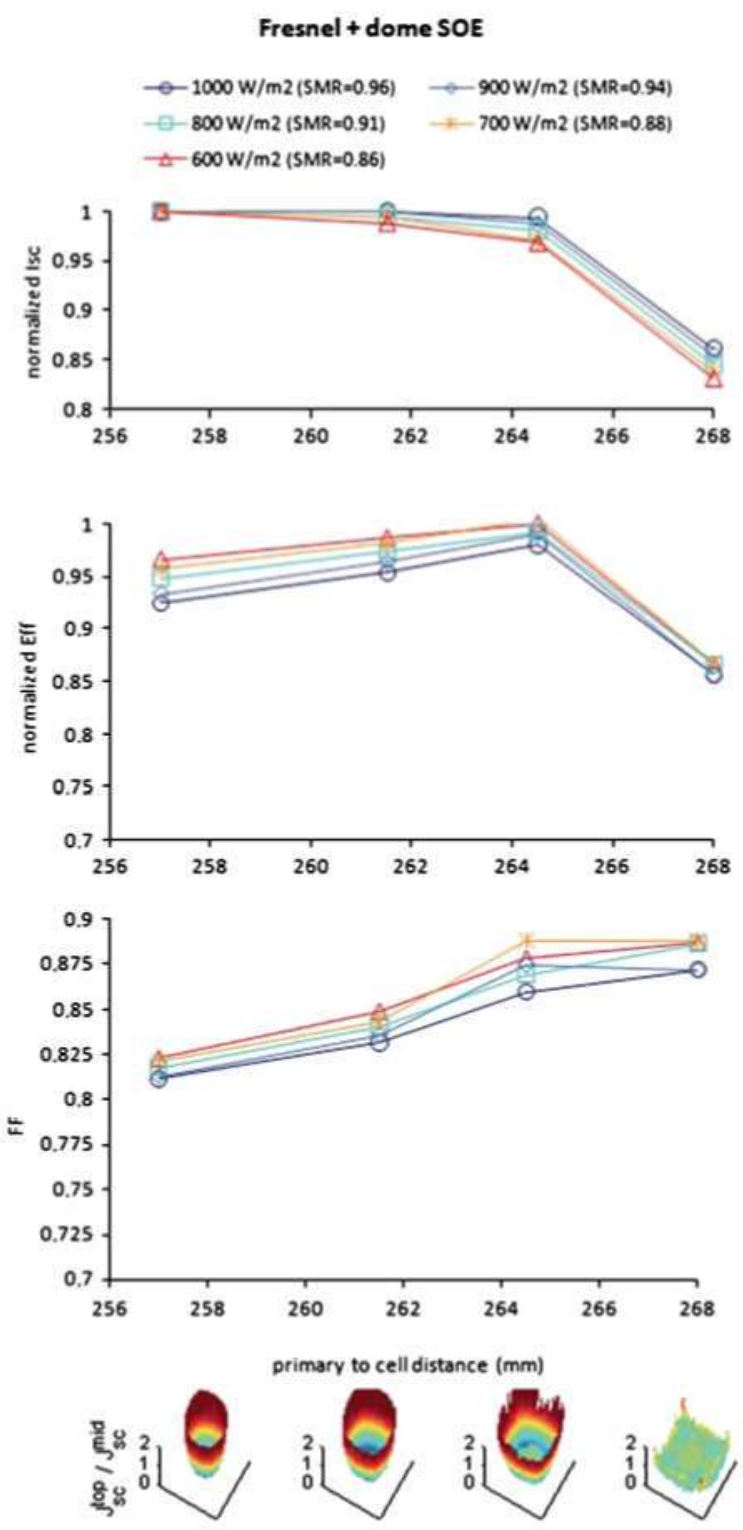

Figure 12. Electrical measurements for the Fresnel+dome secondary optical element concentration system at different cell position. Design position is $265 \mathrm{~mm}$. Plots at the bottom represent ratio between top and middle photogenerated current for each measurement position at $1000 \mathrm{~W} / \mathrm{m}^{2}$ (SMR $\left.=0.96\right)$. Ratios higher than 1 (red) indicate middle-limited zones while ratios lower than 1 (blue) indicate top-limited zones. In green areas;

top and middle photogenerated current is matched.

CPV module in the sun: latitude, time of the day, seasonal variation, and/or atmospheric conditions can easily produce $S M R$ values similar to the ones seen here. This means that a system with an SOE will be less sensitive to the ever-changing incident spectrum in operation, resulting in an increase in the annual energy yield.

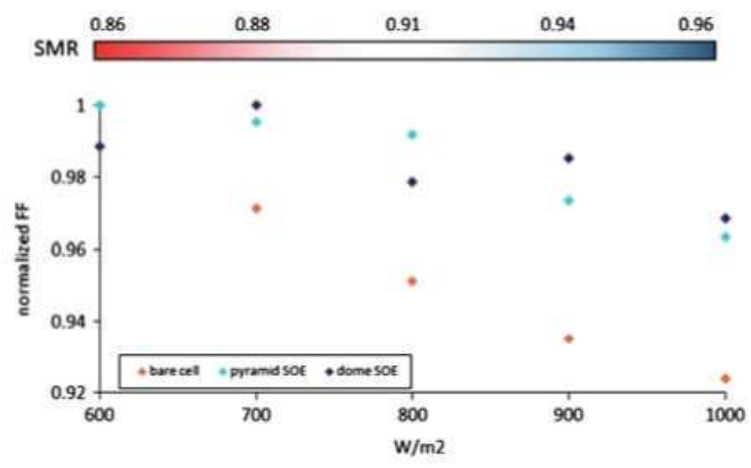

Figure 13. Fill factor (FF) for different irradiance levels $\left(\mathrm{W} / \mathrm{m}^{2}\right.$ ) and spectral matching ratio (SMR) when the cell is at its optimum position in the three optical systems. SOE, secondary optical element.

\section{CONCLUSIONS}

For MJ solar cell performance under a particular CPV system to be accurately predicted, the absolute and spectral spatial non-uniform irradiance profiles created by the system must be studied. In this paper, this has been carried out by both ray-tracing simulations as well as direct measurements.

Comparing three systems, it is shown that adding an SOE to a Fresnel lens significantly reduces those non-uniformities and improves performance. CCD-based measurements show a maximum peak-to-average value of 10 for a Fresnel lens alone, 2 for a Fresnel lens plus pyramid SOE, and 4 for a Fresnel lens plus dome SOE. Ray-tracing simulations predict similar tendencies, but the simulated PAR are higher than those measured by an imaging method due to the absence of scattering in the simulations.

The uniformity enhancement provided by an SOE translates into an efficiency increase. Furthermore, adding an SOE increases tolerance to changes in the incident spectrum. The tolerance improvement will result in an increase in energy yield. The question for designers is whether the extra energy collected compensates for the extra cost of including a secondary element in the optical system. Regardless, for any concentrator system, we have shown that experimental measurements of electrical efficiency versus focal position are fundamental to the selection of the optimum distance to be used for system design, because an incorrect choice will result in lost energy, and simulation is not sufficient to capture real optical behavior.

\section{REFERENCES}

1. Solar junction reaches $43.5 \%$ for CPV. http://www.renewableenergyfocus.com/view/17366/solar-junction-reaches-435efficiency-for-cpv-/ accessed on 30 June 2011. (Archived by WebCite ${ }^{(8)}$ at http://www.webcitation.org/5zpizUH8r)

2. Araki K, Kondo M, Uozumi H, Yamaguchi M. Experimental proof and theoretical analysis on effectiveness of passive homogenizers to $3 \mathrm{~J}$ concentrator solar cells. Proceedings of the 3 rd World Conference 
on Photovoltaic Energy Conversion, 2003; 1: 853 856. DOI: 10.1109/WCPEC.2003.1305417

3. LaRue RA. Borden PG. Gregory PE. A distribuled resistance model of an AlGaAs/GaAs concentrator solar cell illuminated with a curved groove Fresnel lens. IEEE Electron Device Letters 1981; 2: 41-43. DOI: 10.1109/EDL.1981.25333

4. Kurz SR. O'Neill MJ. Estimating and controlling chromatic aberration losses for two-junction, two-terminal devices in refractive concentrator systems. Proceedings of the 25th IEEE Photovolatic Specialists Conference, 1996; 361-364. DOI: 10.1109/PVSC.1996.564020

5. Garcia I, Algora C, Rey-Stolle I, Galiana B. Study of non-uniform light profiles on high concentration solar cells using quasi-3D distributed models. Proceedings of the 33rd IEEE Photovoltaic Specialists Conference, 2008; 1-6. DOI: 10.1109/PVSC. 2008.4922908

6. Nishioka K. Takamoto T. Nakajima W, et al Analysis of triple-junction solar cell under concentration by SPICE. Proceedings of the 3rd World Conference on Photovolraic Energy Conversion. 2003; 869-872. DOI: 10.1109 / WCPEC. 2003.1305421

7. James LW. Effects of concentrator chromatic aberration on multi-junction. Proceedings of the 24th IEEE Photovoltaic Specialists Conference, 1994; 1799-1802. DOI: 10.1109/WCPEC.1994.520652

8. Espinet P, García I. Rey-Stolle I, Algora C. Distribuled simulation of real tunnel junction effects in multjjunction solar cells. 6th International Conference on Concentrating Photovoltaic Systems, 2010.

9. Olson JM. Simulation of current spreading in III-V multi-junction solar cells with non-uniform irradiance. 6th International Conference on Concentrating Photovoltaic Systems. 2010.

10. Wheeldon JF, Valdivia CE, Walker A. et al. GaAs, AlGaAs and InGaP tunnel junctions for multi-junction solar cells under concentration: resistance study. 6 th International Conference on Concentrating Photovoltaic Systems, 2010.

11. Guter W, Bett AW, I-V characterization of devices consisting of solar cells and tunnel diodes. Proceedings of the 4th World Conference on Photovolkaic Energy Conversion. 2006; 749-752. DOI: 10.1109/WCPEC.2006.279564

12. Katz EA, Gordon JM. Feuermann D. Effects of ultrahigh flux and intensity distribution in multi-junction solar cells. Progress in Photovoltaics: Research and Applications 2006; 14: 297-303.

13. Braun A, Hirsch B, Katz EA, Gordon JM. Guter W, Bett AW. Localized irradiation effects on tunnel diode transitions in multi-junction concentrator solar cells. Solar Energy Materials and Solar Cells 2009; 93: 1692-1695.

14. Leutz R, Suzuki A, Akisawa A, Kashiwagi T. Flux uniformity and spectral reproduction on solar concentrators using secondary optics. ISES Solar World Congress, 2001.

15. Winston R. Miñano JC, Benílez P. Nonimaging Oprics. Elsevier Academic Press, 2005.

16. Victoria M, Domínguez C. Antón I, Sala G. Comparative analysis of different secondary optical elements for aspheric primary lenses. Optics Express 2009; 17 : $6487-6492$.

17. Gordon JM. Kalz EA, Tassew W, Feuermann D. Photovoltaic hysteresis and its ramifications for concentrator solar cell design and diagnostics. Applied Physics Letters 2005; 86: 073508.

18. Herrero R. Victoria M. Askins S, et al Indoor characterization of multi-junction solar cells under non uniform light patterns. 6th Intemajional Conference on Concentrating Photovolraic Systems, 2010.

19. Cotal $\mathbf{H}$, Sherif $\mathbf{R}$. The effects of chromatic aberration on the performance of $\mathrm{GaInP} / \mathrm{GaAs} / \mathrm{Ge}$ concentrator solar cells from Fresnel optics. Proceedings of the 31st IEEE Photovolraic Specialists Conference. 2005; 747-750.

20. Kurtz SR. Friedman DJ, Olson JM. The effect of chromatic aberrations on two-junction, two-lerminal. devices on a concentrator system. Proceedings of the 24th IEEE Photovoltaic Specialists Conference 1994; 1791-1794. DOI: $10.1109 /$ WCPEC.1994.520650

21. Herrero R. Victoria M, Domínguez C, Askins S, Antón I. Sala G. CPV optical system imadiance distribution measurements and its effect on nultijunction solar cells. Progress in Photovolagics: Research and Applicarions 2011. DOI: 10. 1002 pip. 1145

22. Dominguez C, Anton I, Sala G. Solar simulator for concentrator photovoltaic systems. Optics Express 2008; 16: 14894-14901.

23. McMahon WE, Emery KE. Friedman DJ. et al. Fill factor as a probe of current-malching for GaInP2/GaAs tandem cells in a concentrator system during outdoor operation. Progress in Photovoltaics: Research and Applications 2008; 16: 213-224.

24. Siefer G, Baur C. Meusel M, Dimroh F. Bett AW. Warta W. Influence of the sinulator spectrum on the calibration of multi-junction solar cells under concentration. Proceedings of the 29h IEEE Photowolaic Specialiss Conference. 2002; 836-839. DOI: 10.1109/PVSC_2002.1190709

25. Ries H, Gordon JM, Lasken M. High-flux photovoltaic solar concentrators with kaleidoscope-based optical designs. Solar Energy 1997; 60: 11-16.

26. Davies PA. Design of single-surface spherical lenses as secondary concentrators for photovoltaic cells. Pure and Applied Optics: Journal of the European Optical Society Part A 1993; 2: 315-324.

27. James $\mathbf{L W}$. Use of imaging refractive secondaries in photovoltaic concentralors. NASA STURecon Technical Report N, 1989: 89. 29833. 